\title{
Presidents Discuss Initiatives
}

EPS initiatives to strengthen physics in east and central Europe (E\&CE) and the former Soviet Union (FSU) have mostly been channelled through the East-West Coordination Committee. Formed in 1989, it focussed on information gathering and dissemination. Such activities are relatively ineffective compared with directly financed programmes such as the extension into E\&CE of the European Mobility Scheme for Physics Students and of the original IBM-supported EARN computer network. Funding from The Soros Foundations for the former was negotiated by N. Króo, the EPS President until his term of office ended at the EPS Council on 24-25 March.

Professor Króo believes that relations between the various parts of Europe are in a new phase where there is a danger of an "economic iron curtain" growing up unless positive steps are taken to correct imbalances. He therefore organized a discussion meeting in February in Budapest with the region's national physical societies to identify priorities and possible new initiatives.

\section{Some General Issues}

The societies' presidents felt that eastwest contacts as reflected by activity connected with mobility, international organizations and the European Commission are fairly healthy. The main concerns lay elsewhere, and many highlighted long-term issues. EPS should work to achieve sound policies for science in the E\&CE and the FSU, such as a commitment by governments to have science included in the European Union's Phare and Tacis programmes.

Governments demonstrate their attitude by rewarding scientific excellence inadequately. This must change otherwise intellectual resources will continue to be lost. Similarly, the promotion of basic research will stimulate young people to stay or to return after training elsewhere.

Participants also noted that physics often enjoyed a privileged position, so physical societies must use conferences and the media to stress the importance of physics in most of today's economies.

\section{Specific Possibilities}

Much can be done at a more microscopic level. Information flow within some countries is still too restricted, and this is reflected by the lack of national policies on information and the slow pace of infrastructure development. It shows up as poor contacts between institutes, thus hindering international cooperation. Collaboration, notably in the field of electronic communications, with outside institutes could lead to dramatic improvements by demonstrating what can be achieved. But one has to be careful in planning collaborations because the cost of expert evaluations to prepare the way often even outweighs support for new equipment.

Information flow has also not been helped by the introduction of market economies. For instance, specialised journals are in short supply, and the cost in Russia of some journals published in Russia is now prohibitively high. Journal exchanges and regional agreements between physical societies can help, as do bilateral agreements of the type the Russian Academy of Sciences has established with FSU countries. These work if communications within the region are well developed. Some agreements include equipment exchange, but in spite of the urgent need for equipment for student laboratories offers of second-hand items should be treated with caution.

The lack, in E\&CE and the FSU, of modern medium-scale facilities such as synchrotrons and neutron sources means that scientists are continually drawn towards the west. Initiatives are therefore needed to stimulate the creation of facilities and centres of excellence. But they must be based on sound proposals for success will ultimately depend on the scientific case.

The region has had little opportunity to remain competitive in international markets for basic, but high value-added, products. In some countries one is in a "destroying period" with industry being stripped of saleable assets. So re-industrialisation will
Meeting of the Presidents of National Physical Societies from East \& Centra Europe \& the Former Soviet Union Budapest, 3-4 February 1995

\section{PARTICIPANTS}

R. Calboreanu, Gen-Sec., Romanian Phys. Soc. M. Furic, Pres., Croatian Phys. Soc. J. Kikas, Pres., Estonian Phys. Soc.

D. Kiss, Pres., Eötvős Lorand Phys. Soc. D. Krupa, Pres., Slovak Physical Society S. Krupica, Pres., Physics Section, Union of Czech Mathematicians \& Physicists N. Króo, Pres., EPS

I. Lalov, Pres., Union of Physicists of Bulgaria N. Mankoc-Borstnik, Pres., Soc. of Mathematicians, Physicists \& Astronomers of Slovenia

Y.A. Ossypian, Russian Academy of Sciences

H. Szymczak, Pres., Polish Phys. Soc.

have to be connected with high-technology. But since there are few physicists left in what remains of industry, EPS will find it difficult to act for them and with them. One has also to be realistic because surveys reveal that industry believes that physics institutes can best help by training young people. Encouraging conferences to be held in the region will promote links to industry, and national societies should play a greater role. A mobility scheme for young people working in industry would be useful.

The mobility of young people is clearly a priority and although much has been achieved, existing initiatives only partly satisfy the demand. Schemes are not yet being fully developed or properly exploited because national physical societies have little experience with them. Their absence for moving within E\&CE and the FSU may explain why it is now much more difficult for a young scientist from say Poland to work in Moscow than in Germany.

The meeting endorsed a suggestion to hold a regular annual meeting of representatives from E\&CE and its neighbours, and an Executive Committee task force headed by N. Króo (who is now the Vice-President for one year) will address east-west issues. Following a suggestion by M. Furic, an immediate task will be to inform the European Union of the region's difficulties with regard to science and technology. It will also take up the challenge that "to cry is not enough, things must be done" by tackling a short-list of specific items which includes:

- Student mobility and journals subscriptions: by seeking additional funding.

- Publications: by promoting Europhysics News as a communication channel.

- The shift of conferences to the region: by entering full-heartedly in their organization.

- Young physicists: by examining a possible extension of the student mobility programme.
President int and representatives of physical societies met with the EPS Kikas, N. Króo, D. Kiss, A. Sileika, D. Krupa, R. Calboreanu, N. MankocBorstnik, H. Szymczak, and S. Krupica. M. Furic is not shown. 